\title{
De las creencias de la comunidad educativa escolar sobre la alimentación, a las políticas institucionales del centro educativo
}

\author{
Perceptions of the educational community on good nutrition and school policies
}

Recibido 31 julio 2014 • Aceptado 20 octubre 2014 • Corregido 24 noviembre 2014

Hilda Patricia Núñez Rivas ${ }^{1}$

Instituto Costarricense de Investigación y

Enseñanza en Nutrición y Salud (INCIENSA)

Cartago, Costa Rica

hnunez@inciensa.sa.cr

Natalia Campos Saborío

Vicerrectoría de Investigación

Universidad Estatal a Distancia (UNED)

San José, Costa Rica

ncampos@uned.ac.cr/nataliacampos07@yahoo.com

Ileana Host Schumacher ${ }^{3}$

Facultad de Microbiología

Universidad de Costa Rica (UCR)

San José, Costa Rica

ileana.holst@ucr.ac.cr

Flory Virginia Alfaro Mora ${ }^{4}$

Instituto Costarricense de Investigación y

Enseñanza en Nutrición y Salud (INCIENSA)

Cartago, Costa Rica

valfaro@inciensa.sa.cr

1 Profesional en Nutrición con Doctorado en Educación, investigadora del INCIENSA y docente de la Universidad Estatal a Distancia (UNED). Miembro de la Comisión Nacional de Lactancia Materna.

2 Profesional en Educación con Doctorado en la rama curricular, docente pensionada de la Universidad de Costa Rica (UCR). Investigadora de la Universidad Estatal a Distancia.

3 Profesional en Microbiología con Maestría Académica en Química Clínica. Docente catedrática de la Facultad de Microbiología e investigadora del Centro de Investigación en Hematologíay Trastornos Afines (CIHATA) de la Universidad de Costa Rica (UCR). Miembro de la Comisión Institucional de Régimen Académico.

4 Profesional en Trabajo Social. Investigadora del Instituto Costarricense de Investigación y Enseñanza en Nutrición y Salud (INCIENSA), Unidad de Salud y Nutrición. 
Resumen. El propósito de este artículo es comprender las creencias sobre alimentación saludable que tiene la comunidad educativa, pues son estas las que rigen las políticas de alimentación en los centros educativos y no los lineamientos técnicos oficiales sobre alimentación escolar, elaborados por el Ministerio de Educación Pública en coordinación con el Ministerio de Salud de Costa Rica. Los hallazgos de este estudio describen las creencias sobre alimentación de los niños y las niñas, expresadas por los miembros del Comité de Salud y Nutrición, el personal docente y el cuerpo administrativo de una escuela, caso de estudio. Asimismo, se presentan las creencias del grupo encargado de cocinar en el comedor estudiantil y de atender la soda, sobre la alimentación que se debe brindar en el comedor estudiantil. Se evidencia que las creencias de la cocinera se constituyen en políticas de alimentación aplicadas en el comedor y en la soda, respecto a la selección de las preparaciones de alimentos y a las porciones que se sirven a los niños y a las niñas, según edad y condición nutricional.

Palabras clave. Creencias; comunidad educativa; alimentación saludable; políticas educativas.

Abstract. This article reviews cultural beliefs at schools surrounding healthy eating habits, since they govern food policies for school lunch programs instead of scientific guidelines established by the Costa Rican Ministry of Public Education and the Ministry of Health. The findings of this study describe cultural beliefs on child nutrition as stated by the Health and Nutrition Committee, teachers, administrative staff at a school - a case study. In addition, the personal opinion of the cafeteria cook tend to become the schools' policies when selecting and preparing the food served in cafeterias as well as the portions based on age and nutritional condition of the child.

Keywords. Belief, educational community, school lunch program, health eating habits, educational policies.

\section{Introducción}

Costa Rica ha experimentado en los últimos 45 años un cambio en el perfil epidemiológico del estado nutricional, siendo notorio la reducción de la prevalencia de desnutrición infantil y el incremento en la prevalencia de sobrepeso y obesidad. En el período 1996-2008/09, la prevalencia de delgadez (IMC < 5 percentil) en escolares (5-12 años), se redujo un 75\% (16.1\% vs 6.1\%), sin que aumentara de forma notoria el porcentaje de escolares con estado nutricional normal (Ministerio de Salud, Instituto Costarricense de Investigación y Enseñanza en Nutrición y Salud, 1996; Ministerio de Salud, Instituto Costarricense de Investigación y Enseñanza en Nutrición y Salud, Caja Costarricense de Seguro Social, Instituto Costarricense sobre Drogas e Instituto Nacional de Estadísticas y Censos, 2010). Para el período de estudio, la prevalencia de escolares con exceso de peso (IMC $\geq 85$ percentil) en el 2008/09 aumentó drásticamente, hasta alcanzar un valor que triplicó la prevalencia observada en 1996 (6.1\% vs. 21.4\%) (Ministerio de Salud et al., 1996; Ministerio de Salud et al., 2010).

La obesidad en estos grupos poblacionales se asocia fundamentalmente a la adopción de un patrón alimentario energéticamente denso y a la escasa actividad física (Vizmanos, Hunot y 
Capdevila, 2006). La evidencia científica señala que la calidad de la dieta decae conforme el niño avanza hacia la adolescencia; la dieta no cumple con la metas nutricionales recomendadas para el buen desarrollo; el consumo de frutas, vegetales, leche y jugos de fruta disminuye, mientras el consumo de alimentos con alto contenido de grasa, azúcar y sodio se incrementa (Golley, Hendrie y McNaughton, 2011), lo que aumenta la posibilidad de los riesgos de obesidad, la diabetes mellitus y enfermedades cardiovasculares en edades tempranas (Holst, Núñez, Monge y Barrantes, 2008; Holst et al., 2009). El estilo de vida del niño, la niña o adolescente depende del desarrollo social y económico del país y de las políticas que se definan en materia de alimentación, educación, agricultura, transporte, planificación urbana, medio ambiente, procesamiento, distribución y comercialización de los alimentos (Ali, Blades, Oates y Blumberg, 2009; Dietz, 2013; Campbell, Pipe y Duhaney, 2014).

El Ministerio de Educación Pública (MEP) y el Ministerio de Salud (MS) han hecho esfuerzos por elaborar políticas y normas referentes a la alimentación en los centros educativos, explicitadas en una serie de documentos: reglamentos, guías de menús y decretos. Sin embargo, hay problemas con su aplicación; la comunidad educativa (docentes, estudiantes, padres y madres de familia, dirección de centros educativos), en su mayoría, no estudia esa documentación, por lo que muchas veces ni siquiera las conocen y por ende, no las aplican. Recientemente, se aprobó el Decreto N036910-MEP-S para el funcionamiento de las sodas escolares en los centros educativos públicos (MEP, 2012), con el fin de normar la alimentación que se oferta, pues se ha comprobado que los productos que expenden son, en su gran mayoría, dañinos para la salud por la cantidad de grasa, azúcares y sal/sodio que contienen.

No obstante, estudios cualitativos acerca de las creencias sobre la obesidad en la niñez escolar de la comunidad educativa (Núñez, 2007; Núñez, Campos, Holst y Alfaro, 2013a) han mostrado hallazgos que señalan a las creencias como sustento de las políticas de alimentación, las cuales se concretan en los quehaceres de la soda y comedor estudiantiles. En la presente investigación se analizan las opiniones que subyacen en las políticas de alimentación que se originan en la comunidad educativa de una escuela urbana pública, las cuales sustentan las decisiones acerca de la alimentación ofrecida en el comedor y la soda estudiantiles.

De ahí, que se estima que las conjeturas en mención, deben conocerse para que las comunidades educativas y la gestión en las instituciones escolares, en particular, tomen conciencia acerca de cómo estas inciden en la alimentación de la población estudiantil y decidir los contenidos y enfoques pedagógicos idóneos, con el fin de fortalecer la construcción de hábitos de vida saludables.

En ese sentido, se requiere que la gestión institucional se enfatice en el mejoramiento de la calidad de la alimentación de la población infanto-juvenil, con un enfoque poblacional, multisectorial, multidisciplinar, adaptado a las circunstancias socioculturales en que los niños y adolescentes se desenvuelven cotidianamente (centro educativo, hogar, barrio/comunidad, 
país) y considerando que, la niñez y la adolescencia son períodos clave para establecer hábitos alimentarios y de vida saludables (Kraak, Story_ y Wartell, 2012).

Finalmente, es de suma importancia que en el marco de la iniciativa de la Red de Políticas Informadas por Evidencias (EVIPNet) ${ }^{5}$, los tomadores de decisiones de los diferentes sectores vinculados, consideren la evidencia científica que este estudio cualitativo aporta para encauzar sus recursos y la gestión hacia el mejoramiento de la alimentación, la capacitación en educación para la salud y la prevención y control de la obesidad en la niñez y la adolescencia.

\section{Referente teórico}

\section{Creencias}

Para Quintana (2001), citada por Núñez et al. (2013b), las creencias son: "un conjunto de realidades, meta-empíricas y de ideas que las personas o grupos, aceptan, reconocen, como principio de cuanto desean pensar, hacer y esperar, en la orientación última de su vida" (p. 3).

Gamborino (2011) señala que la formación de creencias se debe a una serie de razones subjetivas, emocionales y psicológicas, en el contexto de familias, amigos, compañeros de trabajo, la cultura y la sociedad en general, y agrega: "Después de la formación de nuestras creencias, (...) defendemos, justificamos y racionalizamos con una serie de razonamientos intelectuales, argumentos convincentes y explicaciones racionales. Las creencias son lo primero, las explicaciones de las creencias son después" (párr. 4). Partiendo de lo dicho por Gamborino, las creencias se encarnan en el pensamiento y sentimiento de las personas y es difícil erradicarlas, aunque estas no correspondan a una fundamentación científica.

En este estudio de caso interesan las creencias de la comunidad educativa de la escuela, la que se concibe como aquella integrada por estudiantes, docentes, administrativos, padres y madres de familia, así como miembros de la Junta de Educación. Para efectos de esta investigación, entre los administrativos se incluyeron a la concesionaria de la soda y las cocineras del comedor y la soda estudiantiles, pues uno de los ejes del estudio son sus creencias sobre lo que ofrece la escuela en esos establecimientos.

\section{Comunidad educativa}

La comunidad educativa en el Reglamento para el funcionamiento y administración del servicio de soda en los centros educativos públicos (MEP, 2012) está constituida por todas aquellas personas participantes en la educación o que la coadyuvan, en una relación directa o indirecta

5 Para mayor información sobre la EVIPNet, consultar en la siguiente dirección: http://www.paho.org/resscad/images/stories/GUATEMALA/ descargas/CostaRica/informe\%20costa\%20rica\%2020\%20de\%20mayo\%202013\%20final.pdf. 
con el centro educativo. Implica un concepto sociológico dinámico, en el cual intervienen las personas relacionadas con la acción educativa: docentes, estudiantes, padres y madres de familia, personal administrativo (dirección, asistente de dirección, conserjes, guardas, personal de mantenimiento), así como los gobiernos locales y las organizaciones comunales y sociales.

La comunidad educativa se ubica dentro y fuera del centro educativo; se concibe como el escenario donde se realizan las actividades típicas de la educación formal y donde, por tanto, se favorecen procesos de aprendizaje, convivencia y crecimiento individual y social para el logro del bienestar integral de la persona y su calidad de vida. Es un lugar ideal para el desarrollo de acciones de promoción de la salud y prevención de enfermedades, de amplio alcance y repercusión, ya que -como institución social- ejerce gran influencia en los niños, las niñas y en los y las adolescentes en las etapas formativas más importantes de sus vidas, de manera que sus familias y comunidad, en general, también se ven beneficiadas con estas acciones (MEP, 2012).

\section{Políticas educativas sobre alimentación}

En relación con las normas establecidas para brindar la alimentación escolar, estas se encuentran, en el nivel macro, en las políticas públicas de los sectores educación y salud. Las políticas públicas, según Matute, citado por Campos (1997):

(...) constituyen los lineamientos de los programas de gobierno, reflejan las intenciones de las autoridades superiores de gobierno, en cuanto al desarrollo de la salud, de la educación, de la vivienda y de otras áreas de crecimiento del país. Las políticas públicas se convierten en el foco principal de demanda para los miembros de la sociedad. (p. 23)

Respecto a las políticas de alimentación y nutrición de salud pública, Vargas (2011) señala que están orientadas a la población costarricense y se basan en los alimentos y nutrientes que cada persona y población necesita, para el gozo y desarrollo de una sociedad sana. Estas políticas se declaran en la Política de Alimentación y Nutrición (PAN).

En el caso de la política pública de alimentación en el sector educación, Vargas (2011) expone el Programa de Alimentación y Nutrición del Escolar y el Adolescente (PANEA) que establece lineamientos para una alimentación complementaria, de manera que promueva hábitos alimentarios saludables en la población estudiantil, ofrece alimentos nutritivos, refuerza adecuados hábitos de higiene y comportamiento en torno a la alimentación diaria. Es así como, el comedor estudiantil es una valiosa oportunidad para promover los procesos de educación alimentaria nutricional en los educandos (Sánchez, 2005).

En los centros educativos, el MEP trata de organizar y estandarizar los procesos del servicio de comedores, mediante guías de menús balanceados para que los directores y directoras de centros educativos, miembros de las Juntas de Educación y Administrativas, Comité de Salud y 
Nutrición (CSN), así como patronatos escolares u otras asociaciones familiares, los consulten de manera permanente.

De las personas que se relacionan con los servicios de comedor, se destacan los miembros del CSN. Según los lineamientos del PANEA, la misión de estos comités es: "dar apoyo técnico a la institución educativa para el desarrollo de la educación en salud, la educación nutricional y la educación agrícola y para velar por el adecuado funcionamiento de la soda y el comedor estudiantil y/o colegial" (MEP, 2011, p. 19).

Así también, es importante destacar que el reglamento PANEA define que los miembros del CSN tienen el deber de supervisar la alimentación que se vende en la soda. Por lo tanto, los CSN tienen un papel primordial en el cumplimiento de los lineamientos del PANEA en el quehacer, no solo de los comedores sino también de las sodas escolares.

De esta manera, el presente estudio brinda la mirada de lo que realmente sucede en un centro educativo urbano respecto a la alimentación que se ofrece en el comedor y la soda estudiantil. Una realidad escolar que, en el caso de la soda, condujo al MEP y al MS a promulgar el Reglamento para el funcionamiento y administración del servicio de soda en los centros educativos públicos (MEP, 2012), del cual se destacan los considerandos relacionados con la formación sobre la alimentación saludable de los estudiantes, la prevención de la obesidad y de otras enfermedades conexas, así como con los derechos de los estudiantes a tener estilos de vida saludables. En ese sentido, el artículo 15 de este reglamento, prohíbe la venta de bebidas preenvasadas, bocadillos con exceso de grasa y azúcar, bebidas carbonatadas y energéticas, embutidos y otros alimentos cargados de grasa.

Este reglamento es un logro para fomentar estilos de vida saludable en la comunidad educativa y requiere una debida supervisión en su aplicación, de manera que se logren cambios sustantivos en la educación y el consumo de alimentos. De ahí, que se recoge evidencia sobre las creencias acerca de los lineamientos de los centros educativos para regular la alimentación en el comedor y la soda estudiantil, pues las opiniones manifiestas generan políticas de alimentación del centro educativo y en muchos casos, están divorciadas de la normativa técnico-científica: el deber ser de la alimentación saludable en las escuelas.

\section{Metodología}

El estudio es de tipo cualitativo, empleando la metodología etnográfica. Su propósito es evidenciar las políticas alimentarias en los centros educativos, basados en las creencias de la comunidad educativa, principalmente en las de cocineras del comedor y la soda estudiantil, en una escuela urbana y pública de la provincia de San José, Costa Rica.

La ubicación geográfica de esta institución permitió realizar visitas frecuentes y en horarios amplios, de seis horas al día, de tres a cuatro veces a la semana, por aproximadamente 19 meses. 
Esta investigación se enmarca también como estudio de caso, porque su propósito primordial fue comprender, en profundidad, las creencias que subyacen en las políticas de alimentación del centro educativo participante, tanto en los recreos como en la soda y el comedor estudiantil.

Sepresenta la información sistematizada en las macrocategorías: Creencias delos miembros del CSN, docentes y administrativos sobre la alimentación en la escuela; las presunciones de las cocineras sobre la alimentación del comedor estudiantil y la soda.

De acuerdo con el planteamiento de Rodríguez, Gily García (1996), se empleó la observación participante, la entrevista a profundidad, grupos de discusión y la revisión de documentos para la recolección de la información. Asimismo, se analizaron los menús del comedor y de la soda estudiantil de la escuela, y los documentos oficiales sobre la alimentación que debe brindarse en los centros educativos, según PANEA.

Se emplearon instrumentos de distintas tecnologías: cámara fotográfica digitalizadora, la grabadora de audio "tipo periodista", así como hojas en blanco prensadas en una tabla para registrar las notas de campo y las crónicas de observación. Todo ello, con el fin de recolectar en la información, estructuras de significado que informan y testifican los comportamientos de las personas observadas.

Se utilizó el programa ATLAS.ti, (Versión WIN: 5.0.66) para la tabulación de dato, al cual se asignaron 126 documentos (entrevistas y crónicas de observación). Además, se aplicó el programa SPSS para Windows (Versión 20.0) para realizar algunos cálculos estadísticos que complementan el análisis.

El modelo de análisis de datos cualitativos, propuesto por Huberman y Miles (2002), se empleó para la clasificación, despliegue, reducción de datos y extracción o verificación de las conclusiones. La categorización se llevó a cabo tomando en cuenta los criterios temáticos, según la clasificación de Rodríguez et al. (1996). Luego, se procedió a la elaboración de los datos mediante la construcción de matrices. Asimismo, se realizaron lecturas exhaustivas de los datos con el propósito de lograr interpretaciones lo más cercanas a la realidad estudiada, para construir y reconstruir las respuestas a las interrogantes planteadas.

Las estrategias de validación de la información utilizadas fueron: la recolección de los datos escalonadamente (Maxwell, 2002); se inició con los procesos de observación y paulatinamente, se aplicaron las entrevistas en profundidad y los grupos de discusión para profundizar en la temática. Para validar los datos, se empleó el proceso de triangulación de datos por fuentes: observaciones, entrevistas, grupos de discusión, documentos y teoría, de manera que se identificaron patrones, tendencias, contradicciones y ausencias entre categorías y sus derivados.

Así también, se realizaron devoluciones periódicas de los datos a las personas que participaron en el estudio, empleando la técnica de círculos de reflexión que, en su mayoría, se 
aplicó en grupo y, en algunos casos, individualizada. De esta manera, las personas participantes analizaron críticamente la información que se fue recopilando, con el propósito que verificaran si la información era correcta y también tomaran conciencia de sus creencias y prácticas en relación con la temática en estudio.

\section{Resultados}

Los resultados se presentan de acuerdo con las categorías de análisis y sus derivados que se exponen a continuación.

\section{Creencias sobre la alimentación en la escuela, según los miembros del Comité de Salud y Nutrición, docentes y administrativos}

Los resultados del análisis mostraron que esta población tenía sus propias creencias sobre cómo debía ser la alimentación que la escuela brinda a la población estudiantil, para cuidar de su salud.

El personal docente y administrativo del centro educativo en estudio, manifestó no conocer el Manual de menús regionalizados para comedores escolares (MEP y MS, 2004), así como los Lineamientos de Programas de Equidad (MEP, 2012), relacionados con los menús para comedores escolares y el funcionamiento de las sodas escolares y colegiales para Costa Rica. Al respecto, indica una maestra:

"Nos gustaría que si en determinado momento ustedes (las investigadoras) tienen algún tipo de folleto o algún tipo de material donde venga hablando sobre las meriendas, sobre un menú nutritivo y variado para los niños, nos lo hagan saber (...) porque es bueno estar cambiando y a la vez hay recetas que son alimenticias y recetas que no son caras (...)". (Maestra de preescolar, integrante del CSN)

De acuerdo con lo manifiesto por la participante, se puede interpretar que el hecho de que el personal docente y administrativo desconozca los documentos del MEP, los cuales norman la alimentación escolar, indica que hay una alta posibilidad de que las creencias de las personas en mención, estén" definiendo"la calidad y la cantidad de alimentos que brinda la escuela a sus estudiantes.

\section{Las creencias vigentes en el comedor estudiantil y el papel del Comité de Salud y Nutrición}

A lo interno de la escuela en estudio, parte del personal docente y administrativo se organizó y conformó un comité para atender los asuntos relacionados con la alimentación de la población estudiantil, a pesar de que desconocían las directrices del MEP sobre esta temática. Los lineamientos y las actividades relacionados con la alimentación de la población estudiantil son acordados por los miembros del CSN de la escuela, en conjunto con la Junta de Educación. Este comité está integrado por las docentes de los niveles de maternal y preescolar, quienes reciben la colaboración de algunas maestras de la escuela que, tradicionalmente, han formado 
parte de este. Las personas que constituyen dicho comité, afirman conocer su función y tienen la creencia de que son las únicas que deben velar por de la alimentación la población estudiantil en la escuela. Este comité decide aspectos relacionados con la infraestructura del comedor estudiantil, sin que la dirección "de turno" de la escuela tenga una participación activa en el tema. A continuación, los testimonios de varios docentes:

"El Comité de Salud y Nutrición funciona en todas las instituciones y la función que tiene es velar porque en el comedor se dé un buen servicio en todo lo referente a nutrición, a higiene (...)". (Docente de preescolar, integrante del CSN)

"(...) nosotros (se refiere al CSN) hemos venido haciendo cambios en el edificio para mejorarlo (...), el comedor ha ido evolucionando también poco a poco (... .)". "Con la Dirección, es que no, ella atiende otras cosas como los recursos en sumomento queestén en la escuela; usted sabe que las direcciones son de turno, en cambio nosotros no, siempre hemos estado velando, haciendo y deshaciendo con esto del comedory la nutrición, pero para el bien de todos los niños". (Docente de preescolar, integrante del CSN)

Según lo expresado porlas docentes, este comitétienela exclusividad dellevar a cabo el objetivo del CSN y son sus integrantes quienes han hecho historia en su mejoramiento; de esta manera, excluyen a la dirección de su funcionamiento, no porque haya conflictos con la administración del centro educativo, sino porque "las direcciones son de turno" y las maestras tienen continuidad en su labor por años, lo que hace pensar que el manejo del comedor es exclusivo de ellas.

\section{Las normas, las reglas y las prohibiciones son transmitidas verbalmente y la soda "es independiente del comedor"}

Las integrantes del CSN y de la Junta de Educación de la escuela deciden las acciones a seguir en el servicio del comedor estudiantil y los acuerdos (normas, reglas, prohibiciones), relacionados con la alimentación de las niñas y los niños, son transmitidos en forma verbal a las personas vinculadas. Por ejemplo, los acuerdos sobre el servicio de comedor estudiantil son comunicados, mediante una reunión informal mensual o bimensual, a las dos misceláneas que tienen a cargo la preparación, la cocción y la servida de los alimentos en el comedor estudiantil. Esta práctica no es acorde con lo estipulado en la normativa vigente que indica que las comunicaciones deben ser divulgadas mediante circulares.

En relación con las funciones del CSN, sus integrantes tienen la creencia de que les "toca" velar por la alimentación de los y las estudiantes, única y exclusivamente en el comedor estudiantil.

"Ah bueno hay que aclarar que la soda es independiente del comedor estudiantil, porque el comedor estudiantil es financiado por DANEA y la soda es un contrato que se hace con la Junta de Educación. Entonces, con la soda es como un asunto aparte de nosotros, porque a nosotros como Comité nos toca velar por la alimentación del niño, que sea balanceada y todo eso, pero en el comedor". (Docente de preescolar, integrante del CSN) 
"El comedor, me parece que es excelente (...). Ahora, lo de la soda, ya eso pues sí hay que verlo pero es que no hay mucha injerencia; las señoras del comedor o del Comité no tienen nada que ver con la soda". (Docente de Inglés)

La soda no debe ser concebida solo como un negocio. De acuerdo con PANEA, el CSN debe velar no solo por la alimentación que ofrecen las sodas, sino también por las condiciones en que se brinda, función que no está cumpliendo. Desde la perspectiva educativa, la soda no funciona separada del quehacer formador de la escuela, pues está ubicada dentro de ella y es donde consumen alimentos los estudiantes, docentes y el personal administrativo. Por lo tanto, esta se convierte en un espacio educativo para la formación de hábitos en alimentación saludable y el personal del centro educativo, es un vigilante de estos hábitos y del expendio de productos, los que deben estar normados por las autoridades de la institución, de acuerdo con las directrices emanadas por los sectores de educación y salud.

\section{Las creencias de la comunidad educativa sobre la alimentación del comedor estudiantil y la soda}

En esta sección se presentan las tablas, de la uno a la tres, que resumen las creencias de la comunidad educativa, principalmente del personal a cargo de la alimentación en el comedor y la soda estudiantiles.

\section{Tabla 1}

\section{Creencias sobre la oferta de alimentos en el comedor y la soda estudiantiles}

\section{En el comedor estudiantil}

"Bueno, a las señoras del comedor (...) le tomamos parecer a ellas para nos digan de su opinión. (...) nos dicen qué tipo de alimento consideran que le quede..., no tanto más fácil, sino que (...) le quede cómodo hacerlo y que la mayoría de los niños de la escuela lo consuma (...) y también nos acogemos al presupuesto, porque hay meriendas muy ricas, o almuerzos muy ricos pero depende todo de un presupuesto". (Docente de preescolar, integrante del CSN)

"Bueno yo (la cocinera), como decido cómo servir a los niños es que... prácticamente estamos haciendo, no lo que nosotros queremos hacer, ni lo que nos digan sino lo que nosotros vemos que los chiquitos es lo que se comen, porque también puede ser que una sopa con verduras, para nosotros pensaríamos que es más nutritiva, pero para qué la vamos hacer si la vamos a tener que botar, entonces se repiten los platos que ellos a diario comen. Bueno, solo la sopa negra, es una sopa que, que alimenta mucho y a ellos les fascina". (Cocinera a cargo del comedor estudiantil)

\section{En la soda estudiantil}

"Vea qué vende más, pues con la comida chatarra definitivamente se hace más dinero, por ejemplo, hoy pizza vende más que cuando hacemos un almuerzo rico y nutritivo que tiene ensalada, arroz, frijoles, platanito maduro, pollo. Mientras que un almuerzo, rico, almuerzo bueno, 25 almuerzos lo más. $Y$ yo les voy a ofrecer almuerzo y les insisto, mire hay arrocito, frijoles y entonces me dicen:ah no ¿no hay hamburguesas?, ¿yno hay hot dogs? Y con las frutas, tampoco comen (...)". (Encargada de la soda estudiantil) "Buenoel problemaes que hay queverlo no desdeel punto de vista del educador o de la educación sino como de negocio, verdad. La realidad es que la soda es un negocio. O sea quées lo que produce más negocio. Si vos me vas a traer por ejemplo una soda dietética o tipo 'Vishnú' con ensaladas y frutas o algo así, creo que no va a tener el mismo pegue, verdad". (Docente de Inglés)

"(...) es un negocio, porque entonces en la medida que la soda sea rentable el próximo año le aumentan el alquiler y la escuela recibe más dinero y no se está pensando en el niño que está creciendo y haciendo hábitos (....)."(Docente de Español)

Nota: Información suministrada por los participantes en el proceso de investigación. 
De acuerdo con las creencias indicadas en la Tabla 1, la política de preparación y servida de alimentos en el comedor y la soda estudiantiles, se basa en dos criterios de la cocineras: "(...) cómodo de hacerlo y que la mayoría de los niños de la escuela lo consuma (...)" y referente a la soda, que el producto se venda, no importa si es nutritivo o no, pues la soda se concibe como un negocio en donde prima la rentabilidad y no la calidad de la alimentación que se expende (ver Figura 1).

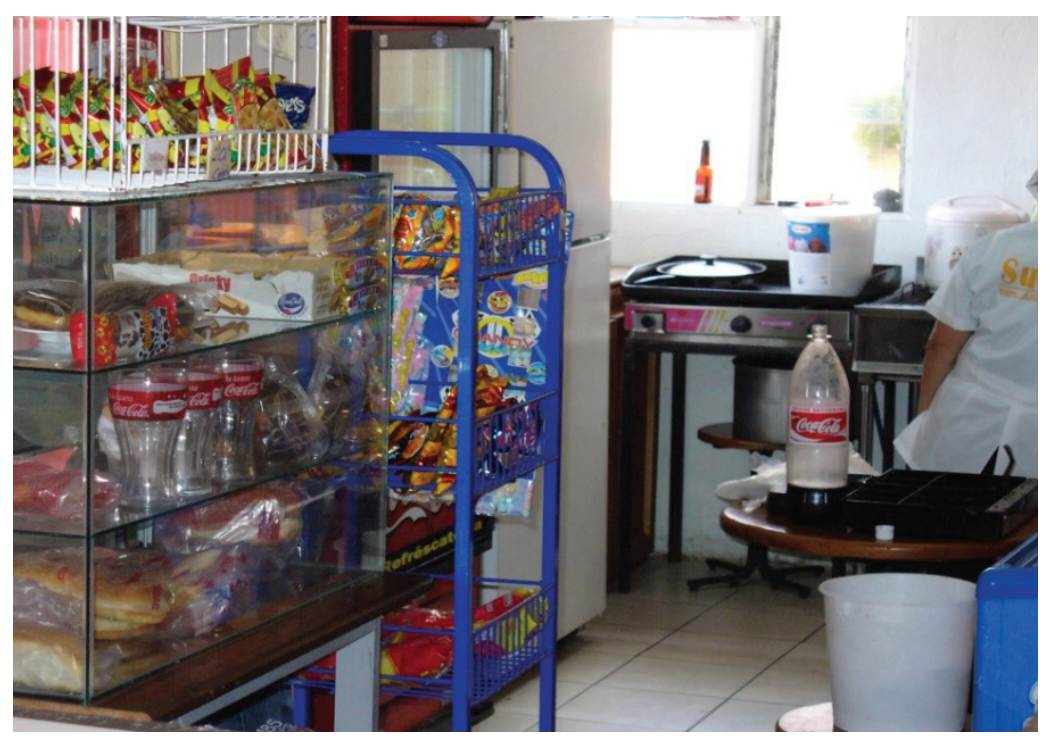

Figura 1. Dispensador de alimentos empacados de la soda estudiantil

Nota: Fotografía autorizada por el centro educativo para efectos de esta investigación.

Por lo tanto, hace falta educar a los niños y niñas, docentes, personal administrativo y padres de familia, en general, sobre alimentación saludable, con el fin de mejorar el servicio que se brinda. Por ejemplo, ahondar en el por qué a los y las estudiantes no les gusta la sopa que no sea de frijol; las frutas y los vegetales. Se debe indagar ¿qué es lo que piensan al respecto?, y no plegarse a lo que a ellos les gusta, pues su gusto por los alimentos puede estar viciado por creencias poco acertadas sobre la alimentación, dada la comunicación masiva existente sobre la comida rápida y otras creencias culturales a las cuales están expuestos cotidianamente. 
Tabla 2

\section{Creencias sobre la porción de alimentos a ofrecer en el comedor y la soda estudiantil}

\begin{tabular}{|c|c|}
\hline En el comedor estudiantil & En la soda estudiantil \\
\hline $\begin{array}{l}\text { "Los gorditos comen más (...) pues les echamos un } \\
\text { poquito más (...) y los de sexto (...) son más grandes } \\
\text { (...) más comelones...están en crecimiento (...) hay } \\
\text { que darles más (...)." (Cocinera a cargo del comedor } \\
\text { estudiantil) } \\
\text { "Es la misma mujer (se refiere a una cocinera del } \\
\text { comedor estudiantil) que hace cuatro años conoce a los } \\
\text { chiquitos; ella sabe aparte de que se ve digamos, el más } \\
\text { gordito o el más flaquito ya ahí se sabe quién come más y } \\
\text { quien no iverdad? entonces aparte de eso, ella los conoce } \\
\text { y entonces este, como hay chiquitos que piden jyo quiero } \\
\text { más! (...) Ahora (...), gordos o flacos, todo se lo comen } \\
\text { (...). (Integrante de la Junta de Educación) } \\
\text { "Bueno, yo sí he visto que algunas veces en el comedor a los } \\
\text { más gorditos les dicen: ¿quiere más? y yo les digo ¿por qué? } \\
\text { y dicen: porque ese come mucho y si come más, pues le } \\
\text { echamos un poquito más". (Docente de Educación Musical) }\end{array}$ & $\begin{array}{l}\text { (...) a la hora ya de servirles (...) me fijo, digamos en } \\
\text { las porciones (...) tomamos en cuenta que los niños } \\
\text { más pequeñitos, se les sirve un poquito menos, porque } \\
\text { inclusive las mismas maestras nos han recomendado } \\
\text { para que haya menos desperdicio, entonces algunas } \\
\text { veces chineamos un poquito más a los más grandecitos } \\
\text { (...), los de quinto y sexto y los de cuarto... que me dicen } \\
\text { iseñora me echa, me da ese que tiene más grande! (...). } \\
\text { (Concesionaria de la soda de la escuela) }\end{array}$ \\
\hline
\end{tabular}

Nota: Información suministrada por los participantes en el proceso de investigación.

Como se interpreta, los criterios que emplean las cocineras para los tipos de alimentos y sus porciones son domésticos, no se fundamentan en información nutricional para la población escolar; aspecto importante a considerar por la administración del centro educativo.

\section{Los menús, su rotación y el presupuesto}

En cuanto a los planes de alimentación y el presupuesto que se asigna a estos, los participantes consideran que:

"(...) el presupuesto por semana son como 700000 más o menos por mes y el menú va en rotación, como que ella (se refiere a la cocinera del comedor estudiantil) tiene un margen de tiempo; por ejemplo, hay una semana en que no se hacen lentejas, ¿verdad? (hay una lista larga donde la rotan, pero (...) la cocinera es la experta en eso. Nosotros ni siquiera vamos a comprar los ingredientes, solo elegimos un proveedor. Las cocineras junto con el Comité de Salud y Nutrición eligen el menú, pues así van pidiendo diario al proveedor; el proveedor trae todos los días la mercadería". (Integrante de la Junta de Educación) 
“(...) cuando vienen las que hacen el menúy todo, ellas (se refiere a las integrantes del Comité de Salud y Nutrición) nos preguntan a nosotros que, qué ponen y nosotros es lo mismo de siempre, aunque los platos se repiten cada quince días. (...) lo que nos piden más (se refiere a los niños y a las niñas) son macarrones (...)". (Encargada de la preparación y la servida de los alimentos en el comedor estudiantil)

"Ylo del presupuesto, bueno eso, esoes muy difícil porquenosotros no, no sabemos exactamente cuánto es el gasto ni nada, pero aquí, digamos lo que yo quiera pedir se puede pedir. Ellas (se refiere a los miembros de la Junta de Educación y del Comité de Nutrición y Salud de la escuela) no le dicen a uno qué se subió o qué se pasó de la cuota ni nada; no, porque supuestamente dicen que DANEA y la Junta se encargan de comprar y de pagar las facturas". (Encargada de la preparación y la servida de los alimentos en el comedor estudiantil)

Del testimonio dado por las personas miembros del CSN, se deduce que este comité es el responsable de: "(...) evaluar y cooperar porque el funcionamiento del comedor sea lo mejor posible, verdad, un éxito (...)". Sin embargo, de los testimonios de las docentes, miembros de la Junta Educativa y del mismo CSN, se determina que son la cocinera y su ayudante quienes deciden qué alimentos y porciones se dan a los estudiantes, tanto en la soda como en el comedor. Sobre este último aspecto, la cocinera también decide sobre la frecuencia con qué se rotan los menús en el comedor. De acuerdo con la evidencia, es imperante la necesidad de capacitar a la comunidad educativa sobre la alimentación escolar que debe ofrecer el centro educativo y las condiciones en las que debe brindarla. 
Tabla 3

Las creencias de la comunidad educativa acerca de la alimentación de los estudiantes el día viernes

\begin{tabular}{|c|c|}
\hline En el comedor estudiantil & En la soda estudiantil \\
\hline $\begin{array}{l}\text { "Yen el comedor también estála cultura de los viernes; los } \\
\text { viernes nunca hay lo que es comida, comida... lo que hay } \\
\text { es o agua dulce con un bollo de pan dulce o chocolate... } \\
\text { a veces les hacen avena... o fruta... o a veces se las } \\
\text { hacen picada y les echan un poquito de... de sirope (...)". } \\
\text { (Docente de cuarto año) } \\
\text { "Los viernes es diferente, porque se hace más liviano } \\
\text { (...). Aquí (se refiere al comedor estudiantil) se les hace } \\
\text { chocolate con algo, pan dulce digamos (...) ah bueno y } \\
\text { también, a veces seles da fruta. Cuando ya llega el viernes, } \\
\text { nosotros como que ya respiramos y porque ya uno se va } \\
\text { de la escuela y es diferente todo y en la escuela también, } \\
\text { porque los maestros, los chiquitos ya (...) estamos como } \\
\text { pensando en el fin de semana (...)." (Encargada de la } \\
\text { preparación y la servida de los alimentos en el comedor } \\
\text { estudiantil) } \\
\text { "Los viernes o los días de celebración ya establecimos por } \\
\text { el MEP, todo se hace un poco diferente, por varios factores: } \\
\text { tiempo, facilidad y población. En el caso del Día del Niño } \\
\text { (...), habían solamente por disposición del Ministerio } 20 \\
\text { minutos para la actividad, } 40 \text { máximo, entonces qué es lo } \\
\text { más fácil, entonces es una situación también de tiempo, } \\
\text { de facilidad y de... para ese tipo de cosas y usted vio, } \\
\text { entonces que se les dio hot dog". (Docente de Inglés) } \\
\text { "Hoy como ya le dije hay perritos calientes y Coca Cola } \\
\text { pues estamos celebrando el Día del Niño, pero por lo } \\
\text { general el menú de aquí es muy nutritivo, ya va usted a } \\
\text { ver". (Directora de la escuela) }\end{array}$ & $\begin{array}{l}\text { "(...) la pizza se quedó solo los viernes, porque... } \\
\text { digamos el viernes es como el día que se pueden } \\
\text { romper las reglas verdad... en el sentido de la comida } \\
\text { (...) los viernes si es diferente al resto de los días (...) } \\
\text { incluso nosotros mismos los maestros también ahí } \\
\text { hacemos algo como diferente". (Docente de cuarto } \\
\text { año) } \\
\text { "Por eso los viernes, se piden } 100 \text { pizzas porque se nos } \\
\text { van todas en el día. Y son de Pizza Hut }{ }^{\circledR} \text {, pequeñas. } \\
\text { Hasta eso, que tiene que ser de esa la pizza porque es } \\
\text { la que a ellos les gusta, verdad". (Encargada de la soda } \\
\text { estudiantil) }\end{array}$ \\
\hline
\end{tabular}

Nota: Información suministrada por los participantes en el proceso de investigación.

Lo citado en los testimonios, evidencia cómo aspectos culturales de la comunidad educativa inciden en los hábitos de alimentación del comedor estudiantil y en las familias de sus estudiantes, pues en los hogares se consume comida rápida durante los fines de semana. Los progenitores de los estudiantes justifican esta costumbre con los mismos criterios de las servidoras del comedor, romper la rutina y la facilidad de cocción de alimentos. Una madre de una niña de cuarto grado dice al respecto: "Los fines de semana, sí... vieras que a veces pedimos pollo... pizza, a veces se nos ocurre comprar así en alguna soda que hay ahí en el barrio de nosotros 
(...)". Así que la política del centro educativo y de los hogares durante los fines de semana, es que seleccionen comidas de fácil preparación, incluyendo la comida "chatarra" para romper la rutina de la semana y descansar de la cocción más elaborada de alimentos.

En la soda se observa que la creencia de la alimentación durante el fin de semana, la ponen en práctica y la constituyen en una política con su repetición en el tiempo. Este aspecto se muestra en el siguiente testimonio y figura:

"Damos pizza los viernes porque tenemos que complacer a todos los chiquitos, ellos nos la piden y los papás les dan a muchos para ese día que son los viernes, para que compren (...). Los papás de ellos, siempre les dan permiso como un día para que coman lo que quieran. Hay un niño que ese día, la mamá lo deja comer chocolates y la pizza también".(Encargada de la soda estudiantil)

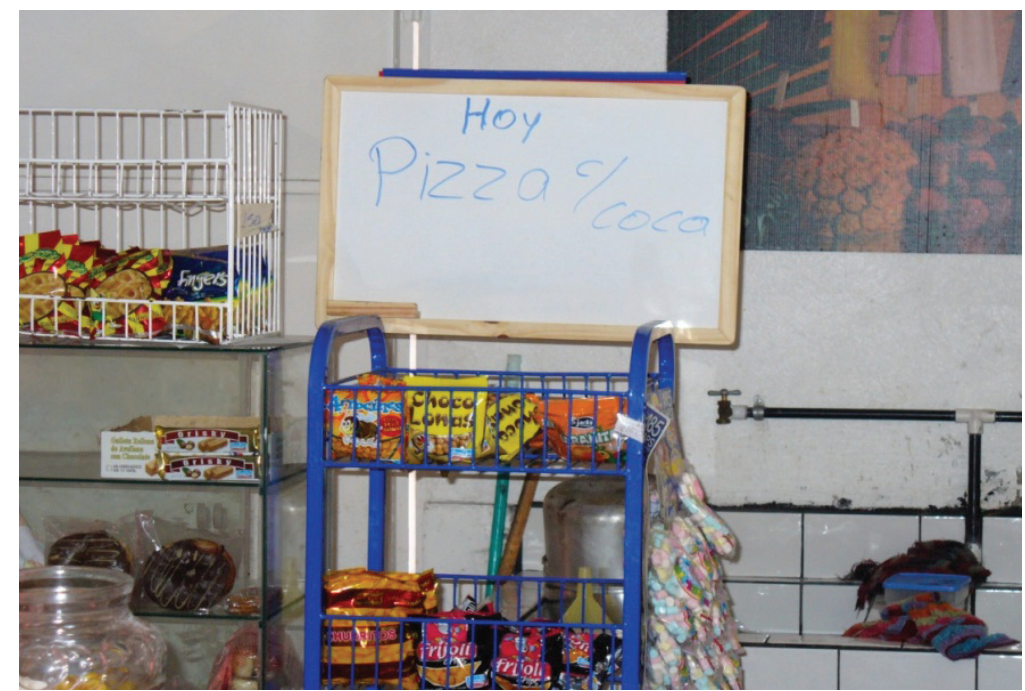

Figura 2. Pizarra acrílica de la soda estudiantil que anuncia que el día viernes se vende pizza con Coca Cola ${ }^{\circledR}$

Nota: Fotografía autorizada por el centro educativo para efectos de esta investigación.

La soda estudiantil no debe ser considerada "un negocio", pues está ubicada en un centro educativo; por lo tanto, su misión debe ser educar para la salud y su oferta alimenticia debe ser supervisada por las autoridades del centro educativo y del sector salud.

Es importante destacar cómo las creencias de la comunidad educativa son las que conforman las políticas de alimentación en la escuela y son estas las que rigen el quehacer escolar en cuanto a la alimentación en el comedor y la soda estudiantil. 


\section{Discusión}

El Ministerio de Educación Pública de Costa Rica procura, en los centros educativos, organizar y estandarizar los procesos del servicio de comedores, mediante guías de menús balanceados para que los directores y directoras, miembros de Juntas de Educación y Administrativas, Comités de Salud y Nutrición, así como patronatos escolares, los consulten de manera permanente. Sin embargo, los resultados de este estudio evidencian que los funcionarios vinculados con el servicio de alimentación estudiantil, no conocen los documentos, de manera que la comunidad educativa asume las políticas de alimentación escolar basadas en las creencias, más que en la documentación técnica y oficial elaborada por el MEP, para tal fin.

EL CSN es la instancia que tiene una gran responsabilidad en vigilar la alimentación estudiantil, pues debe supervisar el desarrollo de la educación para la salud, la nutrición y la agricultura en el centro educativo. Por lo tanto, "velar por el adecuado funcionamiento de la soda y el comedor estudiantil y/o colegial" es una de sus tareas prioritarias (MEP, 2011, p. 20). No obstante, de los testimonios de los miembros del CSN se conoce que: "Las normas, las reglas y las prohibiciones son transmitidas verbalmente". Ello, a pesar de que el artículo 7 del Reglamento del comedor estudiantil indica que el CSN debe: "Levantar actas de los acuerdos tomados en reuniones ordinarias y extraordinarias de este comité" (MEP, 2011, p. 3).

Asimismo, los miembros del CSN informan oralmente a las misceláneas, los acuerdos sobre el servicio del comedor, sin levantar actas como lo solicita el artículo 7 del reglamento en mención. Estos acuerdos son el resultado de las reuniones mensuales o bimensuales que el CSN lleva a cabo sobre el servicio de comedor estudiantil. Acuerdos que no incluyen al servicio de la soda, porque esta no es del resorte del comité y la declaran como "independiente del comedor". No obstante, en el Reglamento del PANEA (MEP, 2011) se expone que el CSN debe vigilar por "el adecuado funcionamiento de la soda" (p. 11). El MEP, mediante algún mecanismo expedito, debe supervisar que la administración y el CSN conozcan los reglamentos y los documentos relacionados con la alimentación que se brinda en los centros educativos, pues, en este caso en estudio, los miembros del CSN no se dan a la tarea de leerlos y opinan que la administración no se ocupa de ello, al comentar que:"(...) las direcciones son de turno (...) nosotros no, siempre hemos estado velando, haciendo y deshaciendo con esto del comedor y la nutrición". Esto, conforme sus formas de pensamiento, porque los miembros del CSN declararon no conocer los reglamentos sobre la alimentación estudiantil.

El hecho de que la administración y el CSN no consulten los documentos del MEP que rigen la alimentación en los centros educativos, puede influir en que sean las creencias de las cocineras las que se convierten en las políticas de alimentación para el comedor estudiantil. Ellas son quienes deciden qué tipos de alimentos seleccionar, qué porciones ofrecer a los estudiantes, cómo rotar lo menús, guiadas por los criterios de servir los alimentos que a los 
estudiantes les gusta, aunque ello implique sustituir los vegetales por las harinas y las comidas rápidas (perros calientes, hamburguesas, pizza, entre otros), principalmente los días viernes. Así lo declara la cocinera cuando dice: "sopa en una mayoría (se refiere a los estudiantes) la botan"; "(...) se les da lo que ellos (los estudiantes) a diario comen".

Lo mencionado anteriormente se opone al artículo del reglamento del comedor estudiantil que señala: "Estimular el desarrollo de actividades especiales dentro de la institución, orientadas al fomento de hábitos alimentarios y de higiene y modales en la población estudiantil" (MEP, 2011, p. 19), pues el CSN y la administración de los centros educativos deben estar claros que su misión no se limita a satisfacer los gustos de los estudiantes en cuanto a la alimentación, sino que deben educarlos sobre la importancia de alimentarse de manera saludable siempre, aunque sea "viernes de moda".

Anteriormente, se señaló que los miembros del CSN no consideraban que fuese su responsabilidad vigilar el funcionamiento de la soda, pues estiman que la soda es un negocio. Al respecto, la encargada de la soda afirma: "La oferta de alimentos de la soda estudiantil (...) comida chatarra definitivamente se hace más dinero (...)". Mientras que el Reglamento de funcionamiento de sodas escolares en centros educativos públicos (Decreto No36910-MEP-S), de reciente aprobación (MEP y MS, 2012), indica:

Que la libertad de comercio que puede darse en los establecimientos o expendios de alimentos dentro de los centros educativos, bajo la figura de concesión de la Junta de Educación o Administrativa, posee una naturaleza mercantil especial de "iure conditio", por lo que es plenamente admisible constituir regulaciones en cuanto a la calidad de los alimentos expendidos, tales como restringir la venta de algunos productos, estableciendo un listado de alimentos adecuados y admisibles para el consumo de la comunidad estudiantil. (p. 10)

Ahora queda la tarea institucional de darle seguimiento a este y otros artículos del Reglamento citado.

Así también, acerca de la soda, un docente dice:"Bueno, el problema es que hay que verlo no desde el punto de vista del educador o de la educación sino como de negocio (Se refiere a la soda), verdad" (Docente de Inglés).

El hecho de que algunos miembros de la comunidad educativa consideren la soda como un negocio, implica que el centro educativo maneja una concepción del estudiante como"cliente consumidor", la cual homogeniza los gustos de la población estudiantil a productos dominantes de la "cultura occidental": coca cola ${ }^{\circledR}$, comida rápida y comida chatarra. Con esta posición, la escuela cosifica a los estudiantes (Barboza, 2012), porque considera que situaciones transitorias son permanentes y no modificables, tal como menciona la concesionaria de la soda: "a todos los niños les gusta el [junk food] y la fruta no (...)"; "ya se intentó y no se vende". Estas declaraciones se presentan como generalizaciones que se aceptan como una realidad dada y absoluta. Con 
estos testimonios, el centro educativo legitima la inserción y permanencia de estos productos en la escuela (ver figuras 1 y 2 ).

Asimismo, se cuestiona a la Junta de Educación sobre una permisión antiética de la escuela. En una reunión con la concesionaria de la soda estudiantil con miembros de la Junta, ella demostró "con los números en la mano", que necesitaba vender esos productos ("chatarra") para obtener suficientes ingresos y así, solventar el pago del alquiler de la soda a la Junta de Educación.

El siguiente discurso de una docente devela con claridad los intereses dominantes de la soda.

"(...) es un negocio, porque entonces en la medida que la soda sea rentable el próximo año le aumentan el alquiler y la escuela recibe más dinero y no se está pensando en el niño que está creciendo y haciendo hábitos, verdad". (Docente de cuarto año)

Queda así demostrado, una vez más, que en el centro educativo estudiado, se priorizan los intereses económicos de la soda, en detrimento de los fines educativos y de la salud integral de la población estudiantil. Además, se debe considerar sus implicaciones en la salud, pues la formación de los hábitos, tanto saludables como no saludables, se inicia desde temprana edad y su modificación es difícil de realizar, porque todo hábito se convierte en una segunda naturaleza (Barrio, 2007); naturaleza que con el hábito se enriquece o se empobrece, se perfecciona o se denigra.

La soda es otro espacio del centro educativo que debe educar en la salud, como lo expresa el artículo 9 del Reglamento mencionado: "Que es obligación del Estado vigilar la salud y el desarrollo integral de los niños, niñasy adolescentes que se encuentran en su etapa formativa yen un período clave de su desarrollo, por lo que se confirma la necesidad de orientar la toma de decisiones de la población estudiantil en relación con la selección de los alimentos saludables" (MEP, 2012, p. 10). Normativa que la comunidad educativa, en general, la administración y el CSN, deben darle riguroso seguimiento para el logro de educar en estilos de vida saludables en el centro educativo.

Así como las cocineras de la soda y del comedor estudiantil, implementan las políticas alimentarias en estos espacios, también proponen otras para la prevención de la obesidad y sobrepeso; al respecto, una de las cocineras afirma: "(...) Aquí se eliminaron las frituras (...) yo (la cocinera) fui la que lo hice (...)". Es una realidad que la alimentación escolar se recarga en las cocineras, situación que debe ser atendida por la comunidad educativa: administrativos (particularmente, el CSN y la dirección del centro educativo), docentes, padres y madres de familia. Una de las cocineras apunta que: "Los papás (...) no les inculcan (...) a los estudiantes comer ensaladas, verduras en el hogar y en el comedor estudiantil no los comen". En consecuencia, el MEP y el MS deben colaborar con la tarea que los padres y madres se formen en educación nutricional, en beneficio de la salud de las familias.

Es indiscutible que la comunidad educativa debe conocer sobre las creencias en lo que se refiere a la alimentación de sus hijos e hijas en el centro educativo, porque estas influyen 
en la conformación de las políticas alimentarias de estas organizaciones, pues algunos centros escolares no regulan la alimentación que se ofrece en las sodas y comedores estudiantiles, según lo estipulado en los respectivos reglamentos, sino que lo hacen de acuerdo con las creencias de la comunidad educativa, primordialmente, las de las cocineras.

El objetivo del centro educativo de hoy, debe ser alcanzar creencias acerca de la obesidad cualitativamente superiores y a la vez, construir las estructuras conceptuales a partir de las creencias de las nuevas generaciones forjadas con los medios masivos de comunicación y no solo a partir del modelo científico, como se ha hecho tradicionalmente en el sistema educativo costarricense.

La Escuela se transforma en un semillero de consumidoras y consumidores habituados, siendo así una aliada de los intereses "ideológicos" de las grandes empresas productoras, como por ejemplo de la trasnacional y mundial "The Coke Cola Company". Este hallazgo es importante destacarlo porque la ideología es producción de sentidos y significados, características que la hacen imprescindible para que la hegemonía pueda actuar y penetrar en todos los rincones y ámbitos de la vida de las niñas y los niños, de las estructuras sociales y del mundo.

Todos esos mecanismos de mercado dan un sentimiento de identidad a los grupos poblacionales (Campbell et al., 2014). La estudiante y el estudiante aprenden en la escuela a ver la identidad como una "identidad de consumo". De ahí, las presiones que ejercen por la disponibilidad de comidas rápidas en la soda estudiantil:

"(...) yo les voy a ofrecer almuerzo y les insisto, mire hay arrocito, frijoles y entonces me dicen: ah no, ¿no hay hamburguesas?, ¿y no hay hot dogs". (Encargada de la soda estudiantil)

Por ello, se considera que estas presiones o reclamos no responden únicamente "al gusto" de las niñas y los niños, sino al significado de esas comidas para el alumnado, cuyo sentido y comunicación se expresa a través de códigos compartidos con sus pares o grupo de pertenencia. Esos códigos se hacen significativos en la medida en que sean sociales y en su socialización intervienen la publicidad, la moda, el consumismo y en general, los medios de comunicación (Kaiser Family Foundation, 2005a; Kaiser Family Foundation, 2005b). Las vivencias de las niñas y los niños se canalizan comercialmente, la expresión de la personalidad se da a través de artículos y productos comprados (Campbell et al., 2014).

Asimismo, en la escuela se busca lo irreflexivo, lo inmediato y lo instantáneo, lo práctico. Tal como lo señalaron algunas de las personas entrevistadas:

"Los viernes o los días de celebración ya establecidos por el MEP, todo se hace un poco diferente, por varios factores: tiempo, facilidad y población, en el caso del Día del Niño, nosotros en una reunión de docentes hablamos; a qué horas por ejemplo va a ser, si se les da de comer arroz o algo que sea más nutritivo a 600 niños en un Día del Niño, cuando había solamente por disposición del Ministerio 20 minutos para la actividad, 40 máximo, entonces qué es lo más 
fácil, entonces es una situación también de tiempo, de facilidad y de... para ese tipo de cosas y usted vio, entonces que se les dio hot dog". (Docente de Inglés)

"Yo creo que uno como padre de la familia, pues parte del estrés es eso, verdad, la carrera, entonces como junk food o la comida chatarra. Y uno envuelve a los chiquillos en eso. Para uno es más fácil pedir una pizza que ponerse a cocinar ensaladas, verduras, que sé yo. Y los chiquillos también, o sea, es parte de la cultura, verdad". (Docente de Inglés)

Al respecto, se considera que la escuela, en estas circunstancias, se encuentra ante un doble desafío. La escuela puede abrirse a este nuevo tipo de saber pragmático y personalizado, útil para la vida práctica y también, acompañar estas nuevas facetas con procesos de reflexión crítica sobre las creencias y las prácticas de alimentación de las niñas, los niños y familias.

Ello, exige que la escuela revise sus enfoques metodológicos y extienda su proyección educativa hacia los hogares de sus estudiantes, pues desde la perspectiva constructivista y del aprendizaje social, es factible llevar a cabo revisiones profundas y críticas de las creencias acerca de la alimentación y lograr el salto cualitativo en estas (Moscovici, Mugny y Pérez, 1991).

Resta acotar que, si en la escuela contemporánea no se dan esos cambios profundos y de revisión permanente, lejos estará el estudiantado de ser formado integralmente, como consumidor crítico y comprometido con su salud individual y la de los suyos, el cuidado de su cuerpo y del medio que le rodea.

\section{Conclusiones}

Las creencias acerca de la alimentación escolar deben ser socializadas en circulares y capacitaciones para el personal docente, administrativo y padres de familia, pues son ellas las que rigen la alimentación de los estudiantes en el comedor y la soda estudiantil. Estas creencias se basan en el sentido común de las personas y, en ocasiones, van en detrimento de la alimentación saludable de los estudiantes.

El Ministerio de Educación Pública debe supervisar que las políticas de alimentación, expresadas en el Reglamento de Comedores Escolares y del Funcionamiento de la Soda Estudiantil, las conozcan y apliquen el personal administrativo y docente, así como las cocineras del comedor y la soda.

La administración escolar debe velar porque los miembros del Comité de Salud y Nutrición ejerzan sus funciones, de acuerdo con lo que establece el PANEA. De lo contrario, son las creencias de las cocineras, las cuales no siempre son las correctas, las que se emplean para la selección, cocción y distribución de los alimentos a los estudiantes.

Se considera un aporte importante de este estudio, el hecho de que las políticas públicas sectoriales de Educación y Salud no impactan el quehacer del centro educativo respecto a la 
alimentación que ofrece, porque son las creencias de la comunidad educativa sobre alimentación y acerca de la prevención de la obesidad, las que rigen el cómo se prepara y cuánto se sirve, causando así una brecha entre lo que técnicamente indican los documentos que se debe hacer y lo que realmente se hace, tanto en el comedor como en la soda estudiantil.

La política institucional de que la soda es un negocio privado, de tipo comida rápida dentro del centro educativo, contradice lo expuesto en el Reglamento para el Funcionamiento de sodas escolares en centros educativos públicos. Por lo tanto, el Comité de Salud y Nutrición, la Junta de Educación y el resto de la comunidad educativa deben intervenir en su quehacer.

La creencia señalada sobre la alimentación para los viernes, evidencia que en la escuela, las niñas y los niños son concebidos como clientes y no como seres humanos en crecimiento y desarrollo integral. Asimismo, sugieren que los progenitores son permisivos con las niñas y los niños, en cuanto al consumo de alimentos tipo "chatarra", por lo que estos también tienen una cuota de contribución importante en la formación de hábitos no saludables de sus hijas e hijos.

\section{Referencias}

Ali, M., Blades, M., Oates, C. \& Blumberg, F. (2009). Young children's ability to recognize advertisements in web page designs. Br J Dev Psycho,_27(Pt 1), 71-83. Recuperado en: http://www.ncbi.nlm.nih.gov/pubmed/19972663

Barboza C., M. C. (2012). Evitando la cosificación del ser humano. Praxis: Relación entre humanos, cómo evitar ser tratados como cosas (poíesis). Revista Iberoamericana para la Investigación y el Desarrollo Educativo, 09. Recuperado en: http://www.ride.org.mx/docs/publicaciones/09/ practica educativa/Ma. Concepcion Barboza Cervantes.pdf

Barrio M., J. M. (2007). Cómo formar la segunda naturaleza. Notas antropológicas acerca de la educación de los hábitos. Estudios sobre Educación, 13, 7-23. Recuperado en: http://dspace. unav.es/dspace/bitstream/10171/9026/1/Ea13.pdf

Campbell, N., Pip, A., \& Duhaney, T. (2014). Calls for restricting the marketing of unhealthy food to children: Canadian cardiovascular health care and scientific community get ignored by policy makers. What can they do? Can J Cardiol, 30(5), 479-81. Doi: 10.1016/j. cjca.2013.11.025. Epub 2013 Dec 4 
Campos S., N. (1997). Política educativa hacia el Siglo XXI: Un análisis de su misión. Revista de Educación, 21(1), 23-28. Recuperado en: http://revistas.ucr.ac.cr/index.php/educacion/ article/view/5466

Dietz, WH. (2013). New strategies to improve food marketing to children. Health Aff (Millwood), 32(9), 1652-8. Doi: 10.1377/hlthaff.2012.1294

Gamborino, P. (2011). Modelos de realidad. Realismo dependiente de Creencias. Parte 1.5. (Post de un blog). Recuperado en: http://blog.mensa.org.mx/756/

Golley, R. K., Hendrie, G. A. \& McNaughton, S. A. (2011). Scores on the dietary guideline index for children and adolescents are associated with nutrient intake and socio-economic position but not adiposity. J Nutr, 141(7), 1340-7. Doi: 10.3945/jn.110.136879

Holst S., I., Núñez R., H., Monge R., R. y Barrantes S., M. (2008). Insulin resistance and impaired glucose tolerance in overweight and obese Costa Rican schoolchildren. Food and Nutrition Bulletin, 29(2), 123-131. Recuperado en: http://europepmc.org/abstract/MED/18693476

Holst S., I., Núñez R., H., Monge R., R. y Barrantes S., M. (2009). Components of the metabolic syndrome among overweight and obese Costa Rican schoolchildren. Food and Nutrition Bulletin, 30(2). Recuperado en: http://www.ingentaconnect.com/content/nsinf/ fnb/2009/00000030/00000002/art00008

Huberman, A. M. y Miles, M. B. (2002). The Qualitative Research's Companion. California, USA: Sage publications Inc.

Kaiser Family Foundation. (2005a). The effects of Electronic Media on Children Ages Zero to Six: A History of Research. Recuperado en: http://kaiserfamilyfoundation.files.wordpress. com/2013/01/the-effects-of-electronic-media-on-children-ages-zero-to-six-a-history-ofresearch-issue-brief.pdf 
Kaiser Family Foundation. (2005b). Executive Summary. Generation M: Media in the Lives of 8-18 Years Old. Recuperado en: http://www.outdoorfoundation.org/pdf/ ExecutiveSummaryGenerationM.pdf

Kraak, Vl., Story, M. \& Wartella, E. A. (2012). Government and school progress to promote a healthful diet to American children and adolescents: a comprehensive review of the available evidence. Am J Prev Med, 42(3), 250-62. Doi: 10.1016/j.amepre.2011.10.025

Maxwell, J. (2002). Understanding Validity in Qualitative Research. En: Huberman, A. M. \& Miles, M. B. The Qualitative Research's Companion (Chapter 2). California, USA: Sage Publications.

Ministerio de Educación Pública. (2011). Programa de alimentación y nutrición del escolar y del adolescente (PANEA). San José, Costa Rica: Autor.

Ministerio de Educación Pública. (2012). Lineamientos de Programas de Equidad. Dirección de Programas de Equidad. Recuperado en: http://www.mep.go.cr/sites/default/files/page/ adjuntos/lineamientos2012.pdf

Ministerio de Educación Pública y Ministerio de Salud. (2004). Manual de menús regionalizados para comedores escolares. Recuperado en: http://www.educatico.ed.cr/Direcciones/DREHH/ Documentos\%20compartidos/DREHH/JUNTAS/MENUS\%20DANEA\%20REGION.pdf

Ministerio de Educación y Ministerio de Salud. (2012). Reglamento para el funcionamiento de sodas escolares en centros educativos públicos. Recuperado en: http://www.gaceta.go.cr/ pub/2012/01/12/ALCA7 1201 2012.pdf

Ministerio de Salud. (1997). Guías alimentarias para la educación nutricional en Costa Rica. San José, Costa Rica: Autor.

Ministerio de Salud. (2003). Política Nacional de Alimentación y Nutrición 2003-2006. San José, Costa Rica: Autor. 
Ministerio de Salud, Instituto Costarricense de Investigación y Enseñanza en Nutrición y Salud. (1996). Encuesta nacional de nutrición. (Fascículo Antropometría). San José, Costa Rica: Autor.

Ministerio de Salud, Instituto Costarricense de Investigación y Enseñanza en Nutrición y Salud, Caja Costarricense de Seguro Social, Instituto Costarricense sobre Drogas e Instituto Nacional de Estadísticas y Censos. (2010). Encuesta nacional de nutrición 2008-2009. (Fascículo Antropometría). San José, Costa Rica: Autor.

Moscovici, S., Mugny, G. y Pérez, J. A. (1991). La influencia social inconsciente. Barcelona: Anthropos.

Núñez R., H. P. (2007). Las creencias sobre obesidad de estudiantes de la educación general básica. Revista Educación, 31(1), 145-164. Recuperado en: http://www.redalyc.org/articulo. oa?id=44031110

Núñez R., H. P., Campos S., N., Holst S., I. y Alfaro M., F. V. (2013a). Las creencias de la docente de educación física sobre la obesidad en la niñez de edad escolar. Revista Electrónica Educare, 17(2), 5-30. Recuperado en: http://www.revistas.una.ac.cr/index.php/EDUCARE/article/ viewFile/5017/4800

Núñez R., H. P., Campos S., N., Alfaro M., F. V. y Holst S., I. (2013b). Las creencias sobre obesidad de niños y niñas en edad escolar y las de sus progenitores. Revista Electrónica Actualidades Investigativas en Educación, 13(2), 1-30. Recuperado en: http://revista.inie. ucr.ac.cr/uploads/tx magazine/creencias-sobre-obesidad-ninos-ninas-edad-escolar-susprogenitores-nunez-campos-alfaro-holst 01.pdf

Quintana, J. M. (2001). Las creencias y la educación. Pedagogía Cosmovisional. Barcelona: Empresa Editorial Herder S.A.

Rodríguez, G., Gil, J. y García, E. (1996). Metodología de la investigación cualitativa. Granada: Ediciones Ajibe. 
Sánchez, F. (2005). Construcción el escenario axiológico para la educación en ambiente y salud en Centros de Educación y Nutrición y Centros Infantiles de Nutrición y Atención Integral (CENCINAI) del Ministerio de Salud. (Tesis de Doctorado, Universidad Estatal a Distancia). Montes de Oca, San José, Costa Rica.

Vargas C., A. (2011). Los programas de alimentación escolar (PAE) en América Latina: una propuesta de modernización para Costa Rica. (Tesis de Doctorado, Universidad Estatal a Distancia). Montes de Oca, San José, Costa Rica. Recuperado en: http://repositorio.uned.ac.cr/reuned/ bitstream/120809/1000/1/Tesis\%20Alexis\%20Vargas.pdf

Vizmanos, B., Hunot, C., Capdevila, F. (2006). Alimentación y obesidad. Investigación en Salud, 8(2), 79-85. Recuperado en: http://www.redalyc.org/pdf/142/14280204.pdf 